PROCEEDINGS OF THE

AMERICAN MATHEMATICAL SOCIETY

Volume 125, Number 1, January 1997, Pages 243-244

S 0002-9939(97)03698-8

\title{
THE ESSENTIAL SELFCOMMUTATOR OF A SUBNORMAL OPERATOR
}

\author{
JOHN B. CONWAY AND NATHAN S. FELDMAN
}

(Communicated by Palle E. T. Jorgensen)

\begin{abstract}
In this paper an easier proof is obtained of Alexandru Aleman's extension of an inequality of Axler and Shapiro for subnormal operators to the essential norm. The method is applied to show that a hyponormal operator whose essential spectrum has area zero must be essentially normal.
\end{abstract}

If $S$ is a subnormal operator on a Hilbert space, it was shown in [4] that the norm of the selfcommutator of $S,\left[S^{*}, S\right]=S^{*} S-S S^{*}$, satisfies the inequality

$$
\left\|S^{*} S-S S^{*}\right\| \leq \operatorname{dist}_{C(\sigma(S))}[\bar{z}, R(\sigma(S))]^{2},
$$

where for any compact subset $K$ of $\mathbb{C}, R(K)$ is the uniform closure in $C(K)$ of the rational functions with poles off $K$. Recently Alexandru Aleman [2] proved a variation on this inequality by calculating the essential norm of $S^{*} S-S S^{*}$; that is, the norm of the image of $S^{*} S-S S^{*}$ in the Calkin algebra. Denote this norm by $\left\|S^{*} S-S S^{*}\right\|_{e}$. He shows that

$$
\left\|S^{*} S-S S^{*}\right\|_{e} \leq \operatorname{dist}_{C\left(\sigma_{e}(S)\right)}\left[\bar{z}, R\left(\sigma_{e}(S)\right)\right]^{2},
$$

where $\sigma_{e}(S)$ is the essential spectrum of $S$.

Aleman's proof uses properties of the positive operator-valued measure associated with $S$ (the compression of the spectral measure of its minimal normal extension ). In this note we give an easier proof of (2) that uses (1) and the fact that a $C^{*}$-algebra has a faithful representation.

The reader might also be cautioned that a study of Aleman's paper is still worth the effort. First, he obtains additional information. Second, the method using the associated positive operator-valued measure seems likely to be useful in attacking additional questions.

These inequalities have a number of important consequences for the study of subnormal operators. When combined with a result from [3], (1) yields Putnam's inequality [7] for subnormal operators:

$$
\left\|S^{*} S-S S^{*}\right\| \leq \frac{\operatorname{Area}(\sigma(S))}{\pi} .
$$

(It should be noted that Putnam's inequality also holds for hyponormal operators. See [6], pages 156 and 176-177.) Inequality (2) shows that if $S$ is a subnormal

Received by the editors August 15, 1995.

1991 Mathematics Subject Classification. Primary 47B20; Secondary 30E10.

Key words and phrases. Subnormal operator, hyponormal operators, essential norm, selfcommutator. 
operator whose essential spectrum has the property that $R\left(\sigma_{e}(S)\right)=C\left(\sigma_{e}(S)\right)$, then $S$ is essentially normal; that is, its selfcommutator is compact. In particular, $S$ is essentially normal if its essential spectrum has area zero.

Let $\mathcal{B}=\mathcal{B}(\mathcal{H})$ denote the algebra of all operators on the separable Hilbert space $\mathcal{H}$ and let $\mathcal{B}_{0}=\mathcal{B}_{0}(\mathcal{H})$ be the algebra of compact operators. Let $\pi: \mathcal{B} \rightarrow \mathcal{B} / \mathcal{B}_{0}$ denote the natural map into the Calkin algebra. So $\sigma_{e}(S)$ is the spectrum of $\pi(S)$ in $\mathcal{B} / \mathcal{B}_{0}$.

Using a result of [5], there is a definition of a subnormal element of a $C^{*}$-algebra. (Also see [6], page 30 and Exercise II.1.2, and a criterion for subnormality from [1].) It follows that if $S$ is a subnormal operator in $\mathcal{B}$, then $\pi(S)$ is subnormal in $\mathcal{B} / \mathcal{B}_{0}$. Let $\mathcal{A}$ be the $C^{*}$-algebra generated by $\pi(S)$ in $\mathcal{B} / \mathcal{B}_{0}$, and let $\rho: \mathcal{A} \rightarrow \mathcal{B}(\mathcal{K})$ be a faithful representation. So $T=\rho(\pi(S))$ is a subnormal operator on $\mathcal{K}$. By (1), $\left\|T^{*} T-T T^{*}\right\| \leq \operatorname{dist}_{C(\sigma(T)}\left(\bar{z}, R(\sigma(T))^{2}\right.$. But $\sigma(T)=\sigma_{e}(S)$ and $\left\|T^{*} T-T T^{*}\right\|=$ $\left\|S^{*} S-S S^{*}\right\|_{e}$. This proves (2).

The same method of proof combined with Putnam's inequality (3) establishes the following result for hyponormal operators.

Theorem. If $S$ is a hyponormal operator, then

$$
\left\|S^{*} S-S S^{*}\right\|_{e} \leq \frac{\operatorname{Area}\left(\sigma_{e}(S)\right)}{\pi} .
$$

Corollary. If $S$ is a hyponormal operator whose essential spectrum has area zero, then $S$ is essentially normal.

\section{REFERENCES}

1. J. Agler, Hypercontractions and subnormality, J Operator Theory 13 (1985), 203-217. MR 86i: 47028

2. A. Aleman, Subnormal operators with compact selfcommutator (preprint).

3. H. Alexander, Projections of polynomial hulls, J. Funct. Analysis 3 (1973), 13-19. MR 49:3209

4. S. Axler and J. Shapiro, Putnam's Theorem, Alexander's spectral area estimate, and VMO, Math. Ann. 271 (1985), 161-183. MR 87b:30053

5. J. W. Bunce and J. A. Deddens, On the normal spectrum of a subnormal operator, Proc. Amer. Math. Soc. 63 (1977), 107-110. MR 55:8863

6. J. B. Conway,, The Theory of Subnormal Operators, Amer. Math. Soc., Providence, 1991. MR 92h:47026

7. C. R. Putnam, An inequality for the area of hyponormal spectra, Math. Zeit. 116 (1970), 323-330. MR 42:5085

Department of Mathematics, University of Tennessee, Knoxville, Tennessee 37996 1300 